Punjab University Journal of Mathematics (2021),53(10),729-739

https://doi.org/10.52280/pujm.2021.531003

\title{
Centre of Unitary Subgroup of Modular Group Algebra's
}

\author{
Saima Parveen ${ }^{1}$, Asia Inam ${ }^{2}$, Farwa Idrees ${ }^{3}$ \\ ${ }^{1,2,3}$ Department of Mathematics, \\ Government College Univeristy, Faisalabad, Pakistan, \\ Email: drsaimaparveen@gcuf.edu.pk ${ }^{1}$, asiainaam9425@gmail.com ${ }^{2}$, \\ farwachattha3044@gmail.com ${ }^{3}$
}

Received: 2 February, 2021 / Accepted: 09 August, 2021 / Published online:26 October, 2021
Abstract.: We establish the structure of the centre of $\mathbb{V}_{*}\left(F_{2^{k}}\left(M_{2^{n+1}}\right)\right)$, $\mathbb{V}_{*}\left(F_{2^{k}}\left(M_{2^{n+1}} \times C_{2}\right)\right)$ and $\mathbb{V}_{*}\left(F_{2^{k}}\left(\left(M_{2^{n+1}} \times C_{2}\right) \times C_{2}\right)\right)$ over a finite field of characteristics 2 where $M_{2^{n+1}}=<\psi, \lambda \mid \psi^{2^{n}}=\lambda^{2}=1, \lambda \psi=$ $\psi^{2^{n+1}} \lambda>$ is the Modular group having order $2^{n+1}$ and $C_{2}$ is a cyclic group of order 2 .

AMS (MOS) Subject Classification Codes: 16S34,15A15, 15A33

Key Words:Modular group, group algebra, circulant matrix, unitary unit group, center of unitary unit group.

\section{INTRODUCTION}

Let $F G$ denote the group algebra of group $G$ over the field $F$. The homomorphism $\Gamma$ : $\mathrm{FG} \rightarrow F$ given by $\Gamma\left(\sum_{h \in G} a_{h} h\right)=\sum_{h \in G} a_{h}$ is called the augmentation mapping of $F G$. Write $U(F G)$ be the unit group of all invertable elements in $F G$ and the normalizes unit group denoted by $V(F G)$ consists of all the invertible elements of $F G$ of augmentation 1. It is well known that $U(F G)=U(F) \times V(F G)$. Let $G$ be a finite $p$-group and $F$ a finite field of characteristic $p$, then the order of $V(F G)$ is $|F|^{|G|-1}$ and $V(F G)$ is a finite p-group. For further details on it see [7]. In 1984 Sandling [10] studied the invertible elements in modular group algebra. This group algebra is of finite abelian p-group and this work contributes a lot in an area namely presentation of group of units. In 1992 Sandling [11] worked on the presentation for unit groups of a modular group algebras of groups of order 16. Bovdi and Sakah [3] studied unitary subgroups of the multiplicative group of a modular group algebra of a finite abelian $p-$ group. This paper gave the solution of problem, posed by S.P.Novikov, on the structure of group $V(F G)$ of group algebra over a finite field having characteristic $\mathrm{p}$.

The anti-automorphism of $\mathrm{FG}$ is the mapping $*: F G \rightarrow F G$ which is defined below

$$
*\left(\sum_{h \in G} \alpha_{h} h\right)=\sum_{h \in G} \alpha_{h} h^{-1}
$$


An element $\eta$ which satisfy $\eta^{-1}=\eta^{*}$, where $\eta$ is an element of normalised unit group, is called unitary element. So unitary unit group of $F G$ is the set of all normalized unit elements that satisfy $\eta^{-1}=\eta^{*}$ and is denoted by $V_{*}(F G)$. In 1994 Bovdi and Kovacs [1] established that $V_{*}\left(F_{2^{k}} G\right)$ is normal in $V_{*}\left(F_{2^{k}} G\right)$ if $\mathrm{G}$ is extraspecial, and studied unitary units of modular group algebra.

In [8] structure of centre of $Z\left(V_{*}\left(F_{2^{m}} M_{16}\right)\right)$ unitary unit subgroup $V_{*}\left(F_{2^{m}} M_{16}\right)$ of group algebra $\left(F_{2^{m}} M_{16}\right)$ is given where

$$
M_{16}=<\psi, \lambda \mid \psi^{8}=\lambda^{2}=1, \lambda \psi=\psi^{5} \lambda>
$$

is modular group of order 16 and $F_{2^{m}}$ is any finite field of characteristic 2 with $2^{m}$ elements. They also described the structure of unitary unit subgroup $V_{*}\left(F_{2^{m}} M_{16}\right)$ of group algebra $F_{2^{m}} M_{16}$. In [9], Raza and Ahmad constructed structure of $V_{*}\left(F_{2^{k}}(Q D)_{16}\right)$ where $(Q D)_{16}$ is known as quasi dihedral group having order 16 . They also described that $\mathrm{Z}\left(V_{*}\left(F_{2^{k}}(Q D)_{16}\right)\right) \cong C_{2}{ }^{4 n}$. We are interested in the structure of the center of unitary unit subgroup of group algebra $\left(F_{2^{k}}\left(\left(M_{2^{n+1}} \times C_{2}\right) \times C_{2}\right)\right)$.

\section{Notations AND Preliminaries}

This section, contains some definitions and results which are very important in our task.

Definition 2.1. Let $R$ be a associative commutative ring with 1 , a ciculant matrix over $R$ is a square $n \times n$ matrix of the form

$$
\operatorname{cir}\left(s_{1}, s_{2}, \ldots s_{n}\right)=\left(\begin{array}{ccccc}
s_{1} & s_{2} & s_{3} & \cdots & s_{n} \\
s_{n} & s_{1} & s_{2} & \cdots & s_{n-1} \\
s_{n-1} & s_{n} & s_{1} & \cdots & s_{n-2} \\
\vdots & \vdots & \vdots & \ddots & \vdots \\
s_{2} & s_{3} & s_{4} & \cdots & s_{1}
\end{array}\right)
$$

where $s_{i} \in R$.

The sum and product of any two circulant matrices is again circulant matrix and is commutable. For further details related to circulant matrices see [4].

Let $\mathrm{G}$ be a finite group and $G=\left\{m_{1}, m_{2}, \ldots ., m_{n}\right\}$, be the fix listing of elements of $\mathrm{G}$ then MG is called matrix of $\mathrm{G}$.

$$
M(G)=\left(\begin{array}{ccccc}
m_{1}^{-1} m_{1} & m_{1}^{-1} m_{2} & m_{1}^{-1} m_{3} & \cdots & m_{1}^{-1} m_{n} \\
m_{2}^{-1} m_{1} & m_{2}^{-1} m_{2} & m_{2}^{-1} m_{3} & \cdots & m_{2}^{-1} m_{n} \\
m_{3}^{-1} m_{1} & m_{3}^{-1} m_{2} & m_{3}^{-1} m_{3} & \cdots & m_{3}^{-1} m_{n} \\
\vdots & \vdots & \vdots & \ddots & \vdots \\
m_{n}^{-1} m_{1} & m_{n}^{-1} m_{2} & m_{n}^{-1} m_{3} & \cdots & m_{n}^{-1} m_{n}
\end{array}\right)
$$


Take any element of RG let say $w \in R G, w=\sum_{t \in G} a_{t} t$ then RG matrix of $\mathrm{w}$ is defined as

$$
M(R G, w)=\left(\begin{array}{ccccc}
a_{t_{1}^{-1} t_{1}} & a_{t_{1}^{-1} t_{2}} & a_{t_{1}^{-1} t_{3}} & \cdots & a_{t_{1}^{-1} t_{n}} \\
a_{t_{2}^{-1} t_{1}} & a_{t_{2}^{-1} t_{2}} & a_{t_{2}^{-1} t_{3}} & \cdots & a_{t_{2}^{-1} t_{n}} \\
a_{t_{3}^{-1} t_{1}} & a_{t_{3}^{-1} t_{2}} & a_{t_{3}^{-1} t_{3}} & \cdots & a_{t_{3}^{-1} t_{n}} \\
\vdots & \vdots & \vdots & \ddots & \vdots \\
a_{t_{n}^{-1} t_{1}} & a_{t_{n}^{-1} t_{2}} & a_{t_{n}^{-1} t_{3}} & \cdots & a_{t_{n}^{-1} t_{n}}
\end{array}\right)
$$

In [6] Hurley introduced the following theorem:

Theorem 2.2. For a given list of elements of group $G$ which has order $n$, there is a bijective ring homomorphism exist between $R G$ and the $n \times n G$-matrices over $R$,

$$
\eta: b \mapsto M(R G, b)
$$

Let $M_{2^{n+1}}=<\psi, \lambda \mid \psi^{2^{n}}=\lambda^{2}=1, \lambda \psi=\psi^{2^{n+1}} \lambda>$ be modular group of finite order $2^{n+1}$ and $F_{2^{k}} M_{2^{n+1}}$ be group algebra having scalars from $F_{2^{k}}$, finite field of characteristics 2. Take arbitrary $v \in V, v=\sum_{\dot{a}=0}^{2^{n}-1} t_{\dot{a}}\left(\left(\psi^{\dot{a}}, 1\right), 1\right)+\sum_{\ddot{a}=0}^{2^{n}-1} u_{\ddot{a}}\left(\left(\psi^{\ddot{a}} \lambda, 1\right), 1\right)+$ $\sum_{\bar{a}=0}^{2^{n}-1} v_{\bar{a}}\left(\left(\psi^{\bar{a}}, \iota\right), 1\right)+\sum_{\tilde{a}=0}^{2^{n}-1} w_{\tilde{a}}\left(\left(\psi^{\tilde{a}} \lambda, \iota\right), 1\right)+\sum_{\breve{a}=0}^{2^{n}-1} \psi_{\breve{a}}\left(\left(\psi^{\check{a}}, 1\right), \iota\right)+\sum_{\hat{a}=0}^{2^{n}-1} \lambda_{\hat{a}}\left(\left(\psi^{\hat{a}} \lambda, 1\right), \iota\right)+$ $\sum_{O=0}^{2^{n}-1} z_{O}\left(\left(\psi^{O}, \iota\right), \iota\right)+\sum_{\dot{a}=0}^{2^{n}-1} s_{a}\left(\left(\psi^{\dot{a}}, \iota\right), \iota\right)$.

Then we have RG-matrix representation of $\mathrm{v}$ as

$$
\sigma(v)=\left(\begin{array}{cccccccc}
\iota_{0} & \iota_{1} & \mu_{0} & \mu_{1} & \psi_{0} & \psi_{1} & \nu_{0} & \nu_{1} \\
\iota_{1}^{\prime} & \iota_{0}^{\prime} & \mu_{1}^{\prime} & \mu_{0}^{\prime} & \psi_{1}^{\prime} & \psi_{0}^{\prime} & \nu_{1} & \nu_{0}^{\prime} \\
\mu_{0} & \mu_{1} & \iota_{0} & \iota_{1} & \nu_{0} & \nu_{1} & \psi_{0} & \psi_{1} \\
\mu_{1}^{\prime} & \mu_{0}^{\prime} & \iota_{1}^{\prime} & \iota_{0}^{\prime} & \nu_{1}^{\prime} & \nu_{0}^{\prime} & \psi_{1}^{\prime} & \psi_{0}^{\prime} \\
\psi_{0} & \psi_{1} & \nu_{0} & \nu_{1} & \iota_{0} & \iota_{1} & \mu_{0} & \mu_{1} \\
\psi_{1}^{\prime} & \psi_{0}^{\prime} & \nu_{1}^{\prime} & \nu_{0}^{\prime} & \iota_{1}^{\prime} & \iota_{0}^{\prime} & \mu_{1}^{\prime} & \mu_{0}^{\prime} \\
\nu_{0} & \nu_{1} & \psi_{0} & \psi_{1} & \mu_{0} & \mu_{1} & \iota_{0} & \iota_{1} \\
\nu_{1}^{\prime} & \nu_{0}^{\prime} & \psi_{1}^{\prime} & \psi_{0}^{\prime} & \mu_{1}^{\prime} & \mu_{0}^{\prime} & \iota_{1}^{\prime} & \iota_{0}^{\prime}
\end{array}\right)
$$

where, $\iota_{0}=\operatorname{circ}\left(t_{0}, t_{1}, t_{2}, t_{3}, \ldots . ., t_{2^{n}-1}\right), \iota_{1}=\operatorname{circ}\left(u_{0}, u_{1}, u_{2}, u_{3}, \ldots \ldots, u_{2^{n}-1}\right)$,

$\mu_{0}=\operatorname{circ}\left(v_{0}, v_{5}, v_{2}, v_{7}, \ldots \ldots, v_{2^{n}-1}\right), \mu_{1}=\operatorname{circ}\left(w_{0}, w_{5}, w_{2}, w_{7}, \ldots \ldots, w_{2^{n}-1}\right)$,

$\psi_{0}=\operatorname{circ}\left(X_{0}, X_{1}, X_{2}, X_{3}, \ldots \ldots, X_{2^{n}-1}\right), \psi_{1}=\operatorname{circ}\left(z_{0}, z_{1}, z_{2}, z_{3}, \ldots . ., z_{2^{n}-1}\right)$,

$\nu_{0}=\operatorname{circ}\left(y_{0}, y_{1}, y_{2}, y_{3}, \ldots . ., y_{2^{n}-1}\right), \nu_{1}=\operatorname{circ}\left(s_{0}, s_{1}, s_{2}, s_{3}, \ldots . ., s_{2^{n}-1}\right)$,

$\iota_{0}^{\prime}=\operatorname{circ}\left(t_{0}, t_{1+z}, t_{2}, t_{3+z}, \ldots . ., t_{2^{n}+z-1}\right), \iota_{1}^{\prime}=\operatorname{circ}\left(u_{0}, u_{1+z}, u_{2}, u_{3+z}, \ldots . ., u_{2^{n}+z-1}\right)$,

$\mu_{0}^{\prime}=\operatorname{circ}\left(v_{0}, v_{1+z}, v_{2}, v_{3+z}, \ldots . ., v_{2^{n}+z-1}\right), \mu_{1}^{\prime}=\operatorname{circ}\left(w_{0}, w_{1+z}\right.$

$\left., w_{2}, w_{3+z}, \ldots . ., w_{2^{n}+z-1}\right)$,

$\psi_{0}^{\prime}=\operatorname{circ}\left(X_{0}, X_{1+z}, X_{2}, X_{3+z}, \ldots . ., X_{2^{n}+z-1}\right), \psi_{1}^{\prime}=\operatorname{circ}\left(z_{0}, z_{1+z^{\prime}}, z_{2}, z_{3+z^{\prime}}, \ldots \ldots, z_{2^{n}+z^{\prime}-1}\right)$,

$\nu_{0}^{\prime}=\operatorname{circ}\left(y_{0}, y_{1+z}, y_{2}, y_{3+z}, \ldots . ., y_{2^{n}+z-1}\right)$ and $\nu_{1}^{\prime}=\operatorname{circ}\left(s_{0}, s_{1+z}, s_{2}, s_{3+z}, \ldots . ., s_{2^{n}+z-1}\right)$

Theorem 2.3. [5] Let $A=\operatorname{circ}\left(t_{1}, t_{2}, t_{3}, \ldots, t_{p^{m}}\right)$, where $t_{i} \in F_{2^{k}}, m \in N_{0}$ and $p$ denotes a prime. Then

$$
A^{p^{m}}=\sum_{j=1}^{p^{m}} t_{i}^{p^{m}} I_{p^{m}} .
$$




\section{Results}

3.1. The Structure of $Z\left(V_{*}\left(F_{2^{k}} M_{2^{n+1}}\right)\right)$.

Theorem 3.2. The center of $Z\left(V_{*}\left(F_{2^{k}} M_{2^{n+1}}\right)\right)$ is isomorphic to $C_{2}^{\left(2^{n-2} .5-1\right) k}$, i.e.

$$
Z\left(V_{*}\left(F_{2^{k}} M_{2^{n+1}}\right)\right) \cong C_{2}^{\left(2^{n-2} \cdot 5-1\right) k} .
$$

Proof. let $\chi=\sum_{\dot{a}=0}^{2^{n}-1} \curlywedge_{\dot{a}} \psi^{\dot{a}}+\sum_{\ddot{a}=0}^{2^{n}-1} \curlyvee_{\ddot{a}} \psi^{\ddot{a}} \lambda$ be an element of $V_{*}\left(F_{2^{k}} M_{2^{n+1}}\right)$ where $\sum_{\dot{a}=0}^{2^{n}-1} \curlywedge_{\dot{a}}=1$ and let $V=V_{*}\left(F_{2^{k}} M_{2^{n+1}}\right)$ for simplicity. Consider the set $C_{v} \psi=\{v \in$ $V: \psi v=v \psi\}$. Now $\psi v-v \psi=0$ if and only if $\psi\left\{\sum_{\dot{a}=0}^{2^{n}-1} \curlywedge_{\dot{a}} \psi^{\dot{a}}+\sum_{\ddot{a}=0}^{2^{n}-1} \Upsilon_{\ddot{a}} \psi^{\ddot{a}} \lambda\right\}-$ $\left\{\sum_{\dot{a}=0}^{2^{n}-1} \curlywedge_{\dot{a}} \psi^{\dot{a}}+\sum_{\ddot{a}=0}^{2^{n}-1} \curlyvee_{\ddot{a}} \psi^{\ddot{a}} \lambda\right\} \psi=0$ which is true if and only if $\Upsilon_{0}=\curlyvee_{2^{n-1}}, \Upsilon_{1}=\curlyvee_{1+2^{n-1}}, \ldots . ., \Upsilon_{2^{n-1}-1}=\Upsilon_{2^{n}-1}$. Therefore,

$$
C_{v} \psi=\left\{\omega=\sum_{\dot{a}=0}^{2^{n}-1} \curlywedge_{\dot{a}} \psi^{\dot{a}}+\sum_{\ddot{a}=0}^{2^{n-1}-1} \Upsilon_{\ddot{a}}\left\{\left(\left(\psi^{\ddot{a}} \lambda+\psi^{\ddot{a}+2^{n-1}} \lambda\right\}\right.\right.\right.
$$

$Z(V)=\left\{\omega \in C_{v} \psi \mid \omega v=v \omega \forall v \in V\right\}$ Take arbitrary $v \in V v=\sum_{\dot{a}=0}^{2^{n}-1} t_{\dot{a}}\left(\left(\psi^{\dot{a}}+\right.\right.$ $\sum_{\ddot{a}=0}^{2^{n}-1} u_{\ddot{a}}\left(\left(\psi^{\ddot{a}} \lambda\right.\right.$ Now $\omega v=v \omega$ if and only if $\Gamma(\omega) \Gamma(v)-\Gamma(v) \Gamma(\omega)=0$

This implies that

$$
\left(\begin{array}{cc}
\jmath & \varpi \\
\varpi & \jmath^{\prime}
\end{array}\right)\left(\begin{array}{cc}
\iota_{0} & \iota_{1} \\
\iota_{1}^{\prime} & \iota_{0}^{\prime}
\end{array}\right)-\left(\begin{array}{cc}
\iota_{0} & \iota_{1} \\
\iota_{1}^{\prime} & \iota_{0}^{\prime}
\end{array}\right)\left(\begin{array}{cc}
\jmath & \varpi \\
\varpi & \jmath^{\prime}
\end{array}\right)=0
$$

$\iota_{0}=\operatorname{circ}\left(t_{0}, t_{1}, t_{2}, t_{3}, \ldots . ., t_{2^{n}-1}\right), \iota_{1}=\operatorname{circ}\left(u_{0}, u_{1}, u_{2}, u_{3}, \ldots . ., u_{2^{n}-1}\right)$,

$\iota_{0}^{\prime}=\operatorname{circ}\left(t_{0}, t_{1+2^{n-1}}, t_{2}, t_{3+2^{n-1}}, \ldots . ., t_{2^{n}+2^{n-1}-1}\right)$,

$\iota_{1}^{\prime}=\operatorname{circ}\left(u_{0}, u_{1+2^{n-1}}, u_{2}, u_{3+2^{n-1}}, \ldots . ., u_{2^{n}+2^{n-1}-1}\right)$,

$\jmath=\operatorname{circ}\left(\curlywedge_{0}, \curlywedge_{1}, \curlywedge_{2}, \curlywedge_{3}, \ldots ., \curlywedge_{2^{n}-1}\right), \varpi=\operatorname{circ}\left(\curlyvee_{0}, \curlyvee_{1}, \curlyvee_{2}, \ldots\right.$,

$\left.\curlyvee_{2^{n-1}-1}, \curlyvee_{0}, \curlyvee_{1}, \curlyvee_{2}, \ldots, \curlyvee_{2^{n-1}-1}\right)$ and $j^{\prime}=\operatorname{circ}\left(\curlywedge_{0}, \curlywedge_{1+2^{n-1}}, \curlywedge_{2}, \curlywedge_{3+2^{n-1}}, \ldots ., \curlywedge_{2^{n}+2^{n-1}-1}\right)$.

Therefore,

which gives

$$
\Gamma(\omega) \Gamma(v)-\Gamma(v) \Gamma(\omega)=0
$$

$$
\curlywedge_{1}=\curlywedge_{1+2^{n-1}}, \curlywedge_{3}=\curlywedge_{3+2^{n-1}}, \ldots . ., \curlywedge_{2^{n-1}-1}=\curlywedge_{2^{n}-1}
$$

Thus, we have centre as follows: $r_{0}+r_{1}\left\{\psi+\psi^{2^{n-1}+1}\right\}+r_{3}\left\{\psi^{3}+\psi^{2^{n-1}+3}\right\}+\ldots .+$ $r_{2^{n-1}-1}\left\{\psi^{2^{n-1}-1}+\psi^{2^{n}-1}\right\}+r_{2} \psi^{2}+r_{4} \psi^{4}+\ldots .+r_{2^{n}-2} \psi^{2^{n}-2}$.

where, $r_{o}=1+r_{2}+r_{4}+\ldots .++r_{2^{n}-2}$. Now we prove that elements of center of $\mathrm{V}$ are also elements of $V_{*}\left(F_{2^{k}} M_{2^{n+1}}\right)$, for this consider an element $m$ from center of $\mathrm{V}$, then

$$
\Gamma(m)=\left(\begin{array}{cc}
\jmath & \varpi \\
\varpi & \jmath_{0}
\end{array}\right)
$$

where the above circulant matrices are defined below

$\jmath=\operatorname{circ}\left(r_{o}, r_{1}, r_{2}, \ldots, r_{2^{n-1}}, r_{1}, r_{2^{n-1}+2} \ldots . ., r_{2^{n-1}-1}\right)$ and

$\varpi=\operatorname{circ}\left(\curlyvee_{o}, \curlyvee_{1}, \curlyvee_{2}, \ldots, \curlyvee_{2^{n-1}-1}, \curlyvee_{o}, \curlyvee_{1}, \curlyvee_{2}, \ldots, \curlyvee_{2^{n-1}-1}\right)$

For unitary element; $m^{*}=m^{-1}$ iff $\Gamma\left(m^{*}\right)=\Gamma\left(m^{-1}\right)$ iff $(\Gamma(m))^{T}=(\Gamma(m))^{-1}$ iff $(\Gamma(m))^{T} \Gamma(m)=I$. 
Consider

$$
\begin{aligned}
(\Gamma(m))(\Gamma(m))^{T} & =\left(\begin{array}{cc}
\jmath & \varpi \\
\varpi & \jmath
\end{array}\right)\left(\begin{array}{cc}
\jmath & \varpi \\
\varpi & \jmath
\end{array}\right) \\
& =\alpha I_{8}
\end{aligned}
$$

because by Theorem 2.3, we have, $\alpha=\jmath^{2}+\varpi^{2}=I$.

$$
=\left(\begin{array}{llllllll}
I & 0 & 0 & 0 & 0 & 0 & 0 & 0 \\
0 & I & 0 & 0 & 0 & 0 & 0 & 0 \\
0 & 0 & I & 0 & 0 & 0 & 0 & 0 \\
0 & 0 & 0 & I & 0 & 0 & 0 & 0 \\
0 & 0 & 0 & 0 & I & 0 & 0 & 0 \\
0 & 0 & 0 & 0 & 0 & I & 0 & 0 \\
0 & 0 & 0 & 0 & 0 & 0 & I & 0 \\
0 & 0 & 0 & 0 & 0 & 0 & 0 & I
\end{array}\right)
$$

So $Z(V) \subset V_{*}\left(F_{2^{k}} M_{2^{n+1}}\right)$ where $F$ is field having characteristic 2 so we have, Hence $C_{2}^{\left(2^{n-2} \cdot 5-1\right) k} \cong Z\left(V_{*}\left(F_{2^{k}} M_{2^{n+1}}\right)\right.$.

\subsection{The Structure of $Z\left(V_{*}\left(F_{2^{k}}\left(M_{2^{n+1}} \times C_{2}\right)\right)\right)$.}

Theorem 3.4. The center of $Z\left(V_{*}\left(F_{2^{k}}\left(M_{2^{n+1}} \times C_{2}\right)\right)\right)$ is isomorphic to $C_{2}^{\left.\left(2^{n-2} .5\right) 2-1\right) k}$, i.e.

$$
C_{2}^{\left.\left(2^{n-2} \cdot 5\right) 2-1\right) k} \cong Z\left(V_{*}\left(F_{2^{k}}\left(M_{2^{n+1}} \times C_{2}\right)\right)\right) .
$$

Proof. $\chi=\sum_{\dot{a}=0}^{2^{n}-1} \curlywedge_{\dot{a}}\left(\psi^{\dot{a}}, 1\right)+\sum_{\ddot{a}=0}^{2^{n}-1} \curlyvee_{\ddot{a}}\left(\psi^{\ddot{a}} \lambda, 1\right)+\sum_{\bar{a}=0}^{2^{n}-1} e_{\bar{a}}\left(\psi^{\bar{a}}, \iota\right)+\sum_{\tilde{a}=0}^{2^{n}-1} f_{\tilde{a}}\left(\psi^{\tilde{a}} \lambda, \iota\right)$ be an element of $V_{*}\left(F_{2^{k}}\left(M_{2^{n+1}} \times C_{2}\right)\right)$ where $\sum_{\dot{a}=0}^{2^{n}-1} \curlywedge_{\dot{a}}+\sum_{\dot{a}=0}^{2^{n}-1} e_{\dot{a}}=1$ and let $V=V_{*}\left(F_{2^{k}}\left(M_{2^{n+1}} \times C_{2}\right)\right)$ for simplicity. Consider the set $C_{v}(\psi, 1)=\{v \in V$ : $(\psi, 1) v=v(\psi, 1)\}$. Now $(\psi, 1) v-v(\psi, 1)=0$ if and only if $(\psi, 1)\left\{\sum_{\dot{a}=0}^{2^{n}-1} \curlywedge_{\dot{a}}\left(\psi^{\dot{a}}, 1\right)+\right.$ $\left.\sum_{\ddot{a}=0}^{2^{n}-1} \curlyvee_{\ddot{a}}\left(\psi^{\ddot{a}} \lambda, 1\right)+\sum_{\bar{a}=0}^{2^{n}-1} e_{\bar{a}}\left(\psi^{\bar{a}}, \iota\right)+\sum_{\tilde{a}=0}^{2^{n}-1} f_{\tilde{a}}\left(\psi^{\tilde{a}} \lambda, \iota\right)\right\}-\left\{\sum_{\dot{a}=0}^{2^{n}-1} \curlywedge_{\dot{a}}\left(\psi^{\dot{a}}, 1\right)+\right.$ $\left.\sum_{\ddot{a}=0}^{2^{n}-1} \Upsilon_{\ddot{a}}\left(\psi^{\ddot{a}} \lambda, 1\right)+\sum_{\bar{a}=0}^{2^{n}-1} e_{\bar{a}}\left(\psi^{\bar{a}}, \iota\right)+\sum_{\tilde{a}=0}^{2^{n}-1} f_{\tilde{a}}\left(\psi^{\tilde{a}} \lambda, \iota\right)\right\}(\psi, 1)=0$

which is true if and only if $\curlyvee_{0}=\curlyvee_{z}, \curlyvee_{1}=\curlyvee_{1+z}, \ldots . ., \curlyvee_{z-1}=\curlyvee_{2^{n}-1}$ and $f_{0}=f_{z}$, $f_{1}=f_{1+z}, \ldots ., f_{z-1}=f_{2^{n}-1}$

where $z=2^{n-1}$. Therefore, $C_{v}(\psi, 1)=\left\{\omega=\sum_{\dot{a}=0}^{2^{n}-1} \curlywedge_{\dot{a}}\left(\psi^{\dot{a}}, 1\right)+\sum_{\bar{a}=0}^{2^{n}-1} e_{\bar{a}}\left(\psi^{\bar{a}}, \iota\right)+\right.$ $\sum_{\ddot{a}=0}^{z-1} \curlyvee_{\ddot{a}}\left\{\left(\psi^{\ddot{a}} \lambda, 1\right)+\left(\psi^{\ddot{a}+z} \lambda, 1\right)\right\}$

$+\sum_{\tilde{a}=0}^{z-1} f_{\tilde{a}}\left\{\left(\psi^{\tilde{a}} \lambda, \iota\right)+\left(\psi^{\tilde{a}+z} \lambda, \iota\right)\right\}$.

$$
Z(v)=\left\{\omega \in C_{v}(\psi, 1) \mid \omega v=v \omega \forall v \in V\right\}
$$

Take arbitrary $v \in V v=\sum_{\dot{a}=0}^{2^{n}-1} t_{\dot{a}}\left(\psi^{\dot{a}}, 1\right)+\sum_{\ddot{a}=0}^{2^{n}-1} u_{\ddot{a}}\left(\psi^{\ddot{a}} \lambda, 1\right)+\sum_{\bar{a}=0}^{2^{n}-1} v_{\bar{a}}\left(\left(\psi^{\bar{a}}, \iota\right), 1\right)+$ $\sum_{\tilde{a}=0}^{2^{n}-1} w_{\tilde{a}}\left(\left(\psi^{\tilde{a}} \lambda, \iota\right), 1\right)$

Now $\omega v=v \omega$ if and only if $\Gamma(\omega) \Gamma(v)-\Gamma(v) \Gamma(\omega)=0$

This implies that 


$$
\begin{gathered}
\left(\begin{array}{cccc}
\jmath_{0} & \varpi_{0} & \jmath_{1} & \varpi_{1} \\
\varpi_{0} & \jmath_{0}^{\prime} & \varpi_{1} & \jmath_{1}^{\prime} \\
\jmath_{1} & \varpi_{1} & \jmath_{0} & \varpi_{0} \\
\varpi_{1} & \jmath_{1}^{\prime} & \varpi_{0} & \jmath_{0}^{\prime}
\end{array}\right)\left(\begin{array}{cccc}
\iota_{0} & \iota_{1} & \mu_{0} & \mu_{1} \\
\iota_{1}^{\prime} & \iota_{0}^{\prime} & \mu_{1}^{\prime} & \mu_{0}^{\prime} \\
\mu_{0} & \mu_{1} & \iota_{0} & \iota_{1} \\
\mu_{1}^{\prime} & \mu_{0}^{\prime} & \iota_{1}^{\prime} & \iota_{0}^{\prime}
\end{array}\right) \\
-\left(\begin{array}{cccc}
\iota_{0} & \iota_{1} & \mu_{0} & \mu_{1} \\
\iota_{1}^{\prime} & \iota_{0}^{\prime} & \mu_{1}^{\prime} & \mu_{0}^{\prime} \\
\mu_{0} & \mu_{1} & \iota_{0} & \iota_{1} \\
\mu_{1}^{\prime} & \mu_{0}^{\prime} & \iota_{1}^{\prime} & \iota_{0}^{\prime}
\end{array}\right)\left(\begin{array}{cccc}
\jmath_{0} & \varpi_{0} & \jmath_{1} & \varpi_{1} \\
\varpi_{0} & \jmath_{0}^{\prime} & \varpi_{1} & \jmath_{1}^{\prime} \\
\jmath_{1} & \varpi_{1} & \jmath_{0} & \varpi_{0} \\
\varpi_{1} & \jmath_{1}^{\prime} & \varpi_{0} & \jmath_{0}^{\prime}
\end{array}\right)=0
\end{gathered}
$$

$\jmath_{0}=\operatorname{circ}\left(\curlywedge_{0}, \curlywedge_{1}, \curlywedge_{2}, \curlywedge_{3}, \ldots . ., \curlywedge_{2^{n}-1}\right), \varpi_{0}=\operatorname{circ}\left(\curlyvee_{0}, \curlyvee_{1}, \curlyvee_{2}, \ldots, \curlyvee_{z-1}, \curlyvee_{0}, \curlyvee_{1}, \curlyvee_{2}, \ldots, \curlyvee_{z-1}\right)$, $\jmath_{1}=\operatorname{circ}\left(e_{0}, e_{1}, e_{2}, e_{3}, \ldots . ., e_{2^{n}-1}\right), \varpi_{1}=\operatorname{circ}\left(f_{0}, f_{1}, f_{2}, \ldots, f_{z-1}, f_{0}, f_{1}, f_{2}, \ldots, f_{z-1}\right)$,

$J_{0}^{\prime}=\operatorname{circ}\left(\curlywedge_{0}, \curlywedge_{1+z}, \curlywedge_{2}, \curlywedge_{3+z}, \ldots . ., \curlywedge_{2^{n}+z-1}\right),,_{1}^{\prime}=\operatorname{circ}\left(e_{0}, e_{1+z}, e_{2}, e_{3+z}, \ldots ., e_{2^{n}+z-1}\right)$,

$\iota_{0}=\operatorname{circ}\left(t_{0}, t_{1}, t_{2}, t_{3}, \ldots . ., t_{2^{n}-1}\right), \iota_{1}=\operatorname{circ}\left(u_{0}, u_{1}, u_{2}, u_{3}, \ldots . ., u_{2^{n}-1}\right)$,

$\mu_{0}=\operatorname{circ}\left(v_{0}, v_{5}, v_{2}, v_{7}, \ldots . ., v_{2^{n}-1}\right), \mu_{1}=\operatorname{circ}\left(w_{0}, w_{5}, w_{2}, w_{7}, \ldots . ., w_{2^{n}-1}\right)$,

$\iota_{1}^{\prime}=\operatorname{circ}\left(u_{0}, u_{1+z}, u_{2}, u_{3+z}, \ldots . ., u_{2^{n}+z-1}\right)$, and $\mu_{1}^{\prime}=\operatorname{circ}\left(w_{0}, w_{1+z}, w_{2}, w_{3+z}, \ldots . ., w_{2^{n}+z-1}\right)$

Therefore,

$$
\Gamma(\omega) \Gamma(v)-\Gamma(v) \Gamma(\omega)=0
$$

then this implies that

$\curlywedge_{1}=\curlywedge_{1+z}, \curlywedge_{3}=\curlywedge_{3+z}, \ldots . ., \curlywedge_{z-1}=\curlywedge_{2^{n-1}} e_{1}=e_{1+z}, e_{3}=e_{3+z}, \ldots . ., e_{z-1}=e_{2^{n-1}}$

Thus, we have centre as follows:

$Z(V)=r_{0}\left((1,1)+r_{1}\left\{(\psi, 1)+\left(\psi^{z+1}, 1\right)\right\}+r_{3}\left\{\left(\psi^{3}, 1\right)+\left(\psi^{z+3}, 1\right)\right\}+\ldots . .+r_{z-1}\left\{\left(\psi^{z-1}, 1\right)+\right.\right.$ $\left.\left(\psi^{2^{n}-1}, 1\right)\right\}+r_{2}\left(\psi^{2}, 1\right)+r_{4}\left(\psi^{4}, 1\right)+\ldots .+r_{2^{n}-2}\left(\psi^{2^{n}-2}, 1\right)+s_{0}(1, \iota)+s_{1}\{(\psi, \iota)+$ $\left.\left(\psi^{z+1}, \iota\right)\right\}+s_{3}\left\{\left(\psi^{3}, \iota\right)+\left(\psi^{z+3}, \iota\right)\right\}+\ldots . .+s_{z-1}\left\{\left(\psi^{z-1}, \iota\right)+\left(\psi^{2^{n}-1}, \iota\right)\right\}+s_{2}\left(\psi^{2}, \iota\right)+$ $s_{4}\left(\psi^{4}, \iota\right)+\ldots+s_{2^{n}-2}\left(\psi^{2^{n}-2}, \iota\right)+\sum_{\dot{a}=0}^{z-1} \curlyvee_{\dot{a}}\left\{\left(\psi^{\dot{a}} \lambda, 1\right)+\left(\psi^{\dot{a}+z} \lambda, 1\right)\right\}+\sum_{\dot{a}=0}^{z-1} f_{\dot{a}}\left\{\left(\psi^{\dot{a}} \lambda, \iota\right)\left(\psi^{\dot{a}+z} \lambda, \iota\right)\right\}$. where, $r_{o}=1+r_{2}+r_{4}+\ldots . .++r_{2^{n}-2}+s_{0}+s_{2}+s_{4}+\ldots . .+s_{2^{n}-2}$. Now we prove that elements of center of $\mathrm{V}$ are also elements of $V_{*}\left(F_{2^{k}}\left(M_{2^{n+1}} \times C_{2}\right)\right)$, for this consider an element $m$ from center of $\mathrm{V}$, then

$$
\Gamma(m)=\left(\begin{array}{cccc}
\jmath_{0} & \varpi_{0} & \jmath_{1} & \varpi_{1} \\
\varpi_{0} & \jmath_{0} & \varpi_{1} & \jmath_{1} \\
\jmath_{1} & \varpi_{1} & \jmath_{0} & \varpi_{0} \\
\varpi_{1} & \jmath_{1} & \varpi_{0} & \jmath_{0}
\end{array}\right)
$$

$\jmath_{0}=\operatorname{circ}\left(r_{o}, r_{1}, r_{2}, \ldots, r_{z}, r_{1}, r_{z+2} \ldots . ., r_{z-1}\right), \jmath_{1}=\operatorname{circ}\left(s_{o}, s_{1}, s_{2}, \ldots ., s_{z}, s_{1}, s_{z+2} \ldots ., s_{z-1}\right)$,

$\varpi_{0}=\operatorname{circ}\left(\curlyvee_{o}, \curlyvee_{1}, \curlyvee_{2}, \ldots . ., \curlyvee_{z-1}, \curlyvee_{0}, \curlyvee_{1}, \curlyvee_{2}, \ldots, \curlyvee_{z-1}\right)$ and

$\varpi_{1}=\operatorname{circ}\left(f_{o}, f_{1}, f_{2}, \ldots . ., f_{z-1}, f_{0}, f_{1}, f_{2}, \ldots ., f_{z-1}\right)$. For unitary element; $m^{*}=m^{-1}$ iff

$\Gamma\left(m^{*}\right)=\Gamma\left(m^{-1}\right)$ iff $(\Gamma(m))^{T}=(\Gamma(m))^{-1}$ iff

$(\Gamma(m))^{T} \Gamma(m)=I$, by using theorem 2.3. So $Z(V) \subset V_{*}\left(F_{2^{k}}\left(M_{2^{n+1}} \times C_{2}\right)\right)$.

Hence

$$
C_{2}^{\left.\left(2^{n-2} \cdot 5\right) 2-1\right) k} \cong Z\left(V_{*}\left(F_{2^{k}}\left(M_{2^{n+1}} \times C_{2}\right)\right)\right)
$$


3.5. The center of $Z\left(V_{*}\left(F_{2^{k}}\left(\left(M_{2^{n+1}} \times C_{2}\right) \times C_{2}\right)\right)\right)$. In this section we describe the center of $Z\left(V_{*}\left(F_{2^{k}}\left(\left(M_{2^{n+1}} \times C_{2}\right) \times C_{2}\right)\right)\right)$.

Lemma 3.6. The center of $Z\left(V_{*}\left(F_{2^{k}}\left(\left(M_{2^{n+1}} \times C_{2}\right) \times C_{2}\right)\right)\right)$ have elements of the form $\left.\left.\left\{r_{0}((1,1), 1)\right\}+r_{1}\{(\psi, 1), 1)+\left(\left(\psi^{z+1}, 1\right), 1\right)\right\}+r_{3}\left\{\left(\psi^{3}, 1\right), 1\right)+\left(\left(\psi^{z+3}, 1\right), 1\right)\right\}+\ldots . .+$ $r_{z-1}\left\{\left(\left(\psi^{z-1}, 1\right), 1\right)+\left(\left(\psi^{2^{n}-1}, 1\right), 1\right)\right\}+r_{2}\left(\left(\psi^{2}, 1\right), 1\right)+r_{4}\left(\left(\psi^{4}, 1\right), 1\right)+\ldots .+r_{2^{n}-2}\left(\left(\psi^{2^{n}-2}, 1\right), 1\right)+$ $\left.\left\{s_{0}((1, \iota), 1)+s_{1}\{(\psi, \iota), 1)+\left(\left(\psi^{z+1}, \iota\right), 1\right)\right\}+s_{3}\left\{\left(\psi^{3}, \iota\right), 1\right)+\left(\left(\psi^{z+3}, \iota\right), 1\right)\right\}+\ldots .$. $s_{z-1}\left\{\left(\left(\psi^{z-1}, \iota\right), 1\right)+\left(\left(\psi^{2^{n}-1}, \iota\right), 1\right)\right\}+s_{2}\left(\left(\psi^{2}, \iota\right), 1\right)+s_{4}\left(\left(\psi^{4}, \iota\right), 1\right)+\ldots+s_{2^{n}-2}\left(\left(\psi^{2^{n}-2}, \iota\right), 1\right)$ $\left.\left\{t_{0}((1, \iota), \iota)+t_{1}\{(\psi, \iota), \iota)+\left(\left(\psi^{z+1}, \iota\right), \iota\right)\right\}+t_{3}\left\{\left(\psi^{3}, \iota\right), \iota\right)+\left(\left(\psi^{z+3}, \iota\right), \iota\right)\right\}+\ldots .+$ $t_{z-1}\left\{\left(\left(\psi^{z-1}, \iota\right), \iota\right)+\left(\left(\psi^{2^{n}-1}, \iota\right), \iota\right)\right\}+t_{2}\left(\left(\psi^{2}, \iota\right), \iota\right)+s_{4}\left(\left(\psi^{4}, \iota\right), \iota\right)+\ldots .+t_{2^{n}-2}\left(\left(\psi^{2^{n}-2}, \iota\right), \iota\right)\left\{u_{0}((1,1), \iota)+\right.$ $\left.\left.u_{1}\{(\psi, 1), \iota)+\left(\left(\psi^{z+1}, 1\right), \iota\right)\right\}+u_{3}\left\{\left(\psi^{3}, 1\right), \iota\right)+\left(\left(\psi^{z+3}, 1\right), \iota\right)\right\}+\ldots . .+u_{z-1}\left\{\left(\left(\psi^{z-1}, 1\right), \iota\right)+\right.$ $\left.\left(\left(\psi^{2^{n}-1}, 1\right), \iota\right)\right\}+u_{2}\left(\left(\psi^{2}, 1\right), \iota\right)+u_{4}\left(\left(\psi^{4}, 1\right), \iota\right)+\ldots+u_{2^{n}-2}\left(\left(\psi^{2^{n}-2}, 1\right), \iota\right)+\sum_{\dot{a}=0}^{z-1} \curlyvee_{\dot{a}}\left\{\left(\left(\psi^{\dot{a}} \lambda, 1\right), 1\right)+\right.$ $\left.\left(\left(\psi^{\dot{a}+z} \lambda, 1\right), 1\right)\right\}+\sum_{\dot{a}=0}^{z-1} f_{\dot{a}}\left\{\left(\left(\psi^{\dot{a}} \lambda, \iota\right), 1\right)\left(\left(\psi^{\dot{a}+z} \lambda, \iota\right), 1\right)\right\}+\sum_{\dot{a}=0}^{z-1} h_{\dot{a}}\left\{\left(\left(\psi^{\dot{a}} \lambda, 1\right), \iota\right)+\right.$ $\left.\left(\left(\psi^{\dot{a}+z} \lambda, 1\right), \iota\right)\right\}+\sum_{\dot{a}=0}^{z-1} J_{\dot{a}}\left\{\left(\left(\psi^{\dot{a}} \lambda, \iota\right), \iota\right)+\left(\left(\psi^{\dot{a}+z} \lambda, \iota\right), \iota\right)\right\}$.

where, $r_{o}=1+r_{2}+r_{4}+\ldots . .++r_{2^{n}-2}+s_{0}+s_{2}+s_{4}+\ldots . .+s_{2^{n}-2}+t_{0}+t_{2}+t_{4}+$ $\ldots . .+t_{2^{n}-2}+u_{0}+u_{2}+u_{4}+\ldots . .+u_{2^{n}-2}$.

Proof. $\chi=\sum_{\dot{a}=0}^{2^{n}-1} \curlywedge_{\dot{a}}\left(\left(\psi^{\dot{a}}, 1\right), 1\right)+\sum_{\ddot{a}=0}^{2^{n}-1} \Upsilon_{\ddot{a}}\left(\left(\psi^{\ddot{a}} \lambda, 1\right), 1\right)$ $+\sum_{\bar{a}=0}^{2^{n}-1} e_{\bar{a}}\left(\left(\psi^{\bar{a}}, \iota\right), 1\right)+\sum_{\tilde{a}=0}^{\eta} f_{\tilde{a}}\left(\left(\psi^{\tilde{a}} \lambda, \iota\right), 1\right)+\sum_{\check{a}=0}^{2^{n}-1} g_{\breve{a}}\left(\left(\psi^{\check{a}}, 1\right), \iota\right)+\sum_{\hat{a}=0}^{2^{n}-1} h_{\hat{a}}\left(\left(\psi^{\hat{a}} \lambda, 1\right), \iota\right)+$ $\sum_{\grave{a}=0}^{2^{n}-1} I_{\dot{a}}\left(\left(\psi^{\grave{a}}, \iota\right), \iota\right)+\sum_{\grave{a}=0}^{2^{n}-1} J_{\grave{a}}\left(\left(\psi^{\grave{a}}, \iota\right), \iota\right)$ be an element of $\left.V_{*}\left(\left(M_{2^{n+1}} \times C_{2}\right) \times C_{2}\right)\right)$ where $\sum_{\dot{a}=0}^{2^{n}-1} \curlywedge_{\dot{a}}+\sum_{\dot{a}=0}^{2^{n}-1} e_{\dot{a}}+\sum_{\dot{a}=0}^{2^{n}-1} I_{\dot{a}}+\sum_{\dot{a}=0}^{2^{n}-1} g_{\dot{a}}=1$ and let $V=V_{*}\left(F_{2^{k}}\left(\left(M_{2^{n+1}} \times\right.\right.\right.$ $\left.\left.\left.C_{2}\right) \times C_{2}\right)\right)$ for simplicity, and $\eta=2^{n}-1$. Consider the set $C_{v}((\psi, 1), 1)=\{v \in$ $V:((\psi, 1), 1) v=v((\psi, 1), 1)\}$. Now $((\psi, 1), 1) v-v((\psi, 1), 1)=0$ if and only if $((\psi, 1), 1)\left\{\left(\sum_{\dot{a}=0}^{2^{n}-1} \curlywedge_{\dot{a}}\left(\left(\psi^{\dot{a}}, 1\right), 1\right)+\sum_{\ddot{a}=0}^{2^{n}-1} \curlyvee_{\ddot{a}}\left(\left(\psi^{\ddot{a}} \lambda, 1\right), 1\right)+\sum_{\bar{a}=0}^{2^{n}-1} e_{\bar{a}}\left(\left(\psi^{\bar{a}}, \iota\right), 1\right)+\right.\right.$ $\sum_{\tilde{a}=0}^{2^{n}-1} f_{\tilde{a}}\left(\left(\psi^{\tilde{a}} \lambda, \iota\right), 1\right)+\sum_{\breve{a}=0}^{2^{n}-1} g_{\check{a}}\left(\left(\psi^{\check{a}}, 1\right), \iota\right)+\sum_{\hat{a}=0}^{2^{n}-1} h_{\hat{a}}\left(\left(\psi^{\hat{a}} \lambda, 1\right), \iota\right)+\sum_{\dot{a}=0}^{2^{n}-1} I_{\dot{a}}\left(\left(\psi^{\dot{a}}, \iota\right), \iota\right)+$ $\left.\left.\sum_{\grave{a}=0}^{2^{n}=1} J_{\grave{a}}\left(\left(\psi^{\grave{a}}, \iota\right), \iota\right)\right)\right\}-\left\{\left(\sum_{\dot{a}=0}^{2^{n}-1} \curlywedge_{\dot{a}}\left(\left(\psi^{\dot{a}}, 1\right), 1\right)+\sum_{\ddot{a}=0}^{2^{n}-1} \curlyvee_{\ddot{a}}\left(\left(\psi^{\ddot{a}} \lambda, 1\right), 1\right)+\sum_{\bar{a}=0}^{2^{n}-1} e_{\bar{a}}\left(\left(\psi^{\bar{a}}, \iota\right), 1\right)+\right.\right.$ $\sum_{\tilde{a}=0}^{2^{n}-1} f_{\tilde{a}}\left(\left(\psi^{\tilde{a}} \lambda, \iota\right), 1\right)+\sum_{\check{a}=0}^{2^{n}-1} g_{\check{a}}\left(\left(\psi^{\breve{a}}, 1\right), \iota\right)+\sum_{\hat{a}=0}^{2^{n}-1} h_{\hat{a}}\left(\left(\psi^{\hat{a}} \lambda, 1\right), \iota\right)+\sum_{\dot{a}=0}^{2^{n}-1} I_{\dot{a}}\left(\left(\psi^{\dot{a}}, \iota\right), \iota\right)+$ $\left.\left.\sum_{\grave{a}=0}^{2^{n}}-1 J_{\grave{a}}\left(\left(\psi^{\grave{a}}, \iota\right), \iota\right)\right)\right\}$

$((\psi, 1), 1)$.

which is true if and only if $\Upsilon_{0}=\Upsilon_{z}, \Upsilon_{1}=\Upsilon_{1+z}, \ldots ., \Upsilon_{z-1}=\Upsilon_{2^{n}-1}, f_{0}=f_{z}$, $f_{1}=f_{1+z}, \ldots ., f_{z-1}=f_{2^{n}-1}, h_{0}=h_{z}, h_{1}=h_{1+z}, \ldots ., h_{z-1}=h_{2^{n-1}}$ and $J_{0}=J_{z}$, $J_{1}=J_{1+z}, \ldots ., J_{z-1}=J_{2^{n}-1}$ where $z=2^{n-1}$.

Therefore, $C_{v}((\psi, 1), 1)=\left\{\omega=\sum_{\dot{a}=0}^{2^{n}-1} \curlywedge_{\dot{a}}\left(\left(\psi^{\dot{a}}, 1\right), 1\right)+\sum_{\bar{a}=0}^{2^{n}-1} e_{\bar{a}}\left(\left(\psi^{\bar{a}}, \iota\right), 1\right)+\sum_{\check{a}=0}^{2^{n}-1} g_{\breve{a}}\left(\left(\psi^{\breve{a}}, 1\right), \iota\right)+\right.$ $\sum_{\dot{a}=0}^{2^{n}-1} I_{\dot{a}}\left(\left(\psi^{\dot{a}}, \iota\right), \iota\right)+\sum_{\ddot{a}=0}^{z-1} \Upsilon_{\ddot{a}}\left\{\left(\left(\psi^{\ddot{a}} \lambda, 1\right), 1\right)+\left(\left(\psi^{\ddot{a}+z} \lambda, 1\right), 1\right)\right\}+\sum_{\tilde{a}=0}^{z-1} f_{\tilde{a}}\left\{\left(\left(\psi^{\tilde{a}} \lambda, \iota\right), 1\right)+\right.$ $\left.\left(\left(\psi^{\tilde{a}+z} \lambda, \iota\right), 1\right)\right\}+\sum_{\hat{a}=0}^{z-1} h_{\hat{a}}\left\{\left(\left(\psi^{\hat{a}} \lambda, 1\right), \iota\right)+\left(\left(\psi^{\hat{a}+z} \lambda, 1\right), \iota\right)\right\}+\sum_{\grave{a}=0}^{z-1} J_{\grave{a}}\left\{\left(\left(\psi^{\grave{a}} \lambda, \iota\right), \iota\right)+\right.$ $\left.\left.\left(\left(\psi^{\grave{a}+z} \lambda, \iota\right), \iota\right)\right\}\right\}$. Since center of "V" is a subset of centralizer, therefore we have

$$
Z(v)=\left\{\omega \in C_{v}((\psi, 1), 1) \mid \omega v=v \omega \forall v \in V\right\}
$$

Take arbitrary $v \in V$ as follows $v=\sum_{\dot{a}=0}^{2^{n}-1} t_{\dot{a}}\left(\left(\psi^{\dot{a}}, 1\right), 1\right)+\sum_{\ddot{a}=0}^{2^{n}-1} u_{\ddot{a}}\left(\left(\psi^{\ddot{a}} \lambda, 1\right), 1\right)+\sum_{\bar{a}=0}^{2^{n}-1} v_{\bar{a}}\left(\left(\psi^{\bar{a}}, \iota\right), 1\right)+\sum_{\tilde{a}=0}^{2^{n}-1} w_{\tilde{a}}\left(\left(\psi^{\tilde{a}} \lambda, \iota\right), 1\right)+$
$\sum_{\breve{a}=0}^{2^{n}-1} \psi_{\breve{a}}\left(\left(\psi^{\check{a}}, 1\right), \iota\right)+\sum_{\hat{a}=0}^{2^{n}-1} \lambda_{\hat{a}}\left(\left(\psi^{\hat{a}} \lambda, 1\right), \iota\right)+\sum_{O=0}^{2^{n}-1} z_{O}\left(\left(\psi^{O}, \iota\right), \iota\right)+\sum_{\dot{a}=0}^{2^{n}-1} s_{a}\left(\left(\psi^{a}, \iota\right), \iota\right)$. 
Now $\omega v=v \omega$ if and only if $\Gamma(\omega) \Gamma(v)-\Gamma(v) \Gamma(\omega)=0$

This implies that

$$
\begin{aligned}
& \left(\begin{array}{cccccccc}
\jmath_{0} & \varpi_{0} & \jmath_{1} & \varpi_{1} & \jmath_{2} & \varpi_{2} & \jmath_{3} & \varpi_{3} \\
\varpi_{0} & \jmath_{0}^{\prime} & \varpi_{1} & \jmath_{1}^{\prime} & \varpi_{2} & \jmath_{2}^{\prime} & \varpi_{3} & \jmath_{3}^{\prime} \\
\jmath_{1} & \varpi_{1} & \jmath_{0} & \varpi_{0} & \jmath_{3} & \varpi_{3} & \jmath_{2} & \varpi_{2} \\
\varpi_{1} & \jmath_{1}^{\prime} & \varpi_{0} & \jmath_{0}^{\prime} & \varpi_{3} & \jmath_{3}^{\prime} & \varpi_{2} & \jmath_{2}^{\prime} \\
\jmath_{2} & \varpi_{2} & \jmath_{3} & \varpi_{3} & \jmath_{0} & \varpi_{0} & \jmath_{1} & \varpi_{1} \\
\varpi_{2} & \jmath_{2}^{\prime} & \varpi_{3} & \jmath_{3}^{\prime} & \varpi_{0} & \jmath_{0} & \varpi_{1} & \jmath_{1}^{\prime} \\
\jmath_{3} & \varpi_{3} & \jmath_{2} & \varpi_{2} & \jmath_{1} & \varpi_{1} & \jmath_{0} & \varpi_{0} \\
\varpi_{3} & \jmath_{3}^{\prime} & \varpi_{2} & \jmath_{2}^{\prime} & \varpi_{1} & \jmath_{1}^{\prime} & \varpi_{0} & \jmath_{0}^{\prime}
\end{array}\right)\left(\begin{array}{cccccccc}
\iota_{0} & \iota_{1} & \mu_{0} & \mu_{1} & \psi_{0} & \psi_{1} & \nu_{0} & \nu_{1} \\
\iota_{1}^{\prime} & \iota_{0}^{\prime} & \mu_{1}^{\prime} & \mu_{0}^{\prime} & \psi_{1}^{\prime} & \psi_{0}^{\prime} & \nu_{1} & \nu_{0}^{\prime} \\
\mu_{0} & \mu_{1} & \iota_{0} & \iota_{1} & \nu_{0} & \nu_{1} & \psi_{0} & \psi_{1} \\
\mu_{1}^{\prime} & \mu_{0}^{\prime} & \iota_{1}^{\prime} & \iota_{0}^{\prime} & \nu_{1}^{\prime} & \nu_{0}^{\prime} & \psi_{1}^{\prime} & \psi_{0}^{\prime} \\
\psi_{0} & \psi_{1} & \nu_{0} & \nu_{1} & \iota_{0} & \iota_{1} & \mu_{0} & \mu_{1} \\
\psi_{1}^{\prime} & \psi_{0}^{\prime} & \nu_{1}^{\prime} & \nu_{0}^{\prime} & \iota_{1}^{\prime} & \iota_{0}^{\prime} & \mu_{1}^{\prime} & \mu_{0}^{\prime} \\
\nu_{0} & \nu_{1} & \psi_{0} & \psi_{1} & \mu_{0} & \mu_{1} & \iota_{0} & \iota_{1} \\
\nu_{1}^{\prime} & \nu_{0}^{\prime} & \psi_{1}^{\prime} & \psi_{0}^{\prime} & \mu_{1}^{\prime} & \mu_{0}^{\prime} & \iota_{1}^{\prime} & \iota_{0}^{\prime}
\end{array}\right)
\end{aligned}
$$

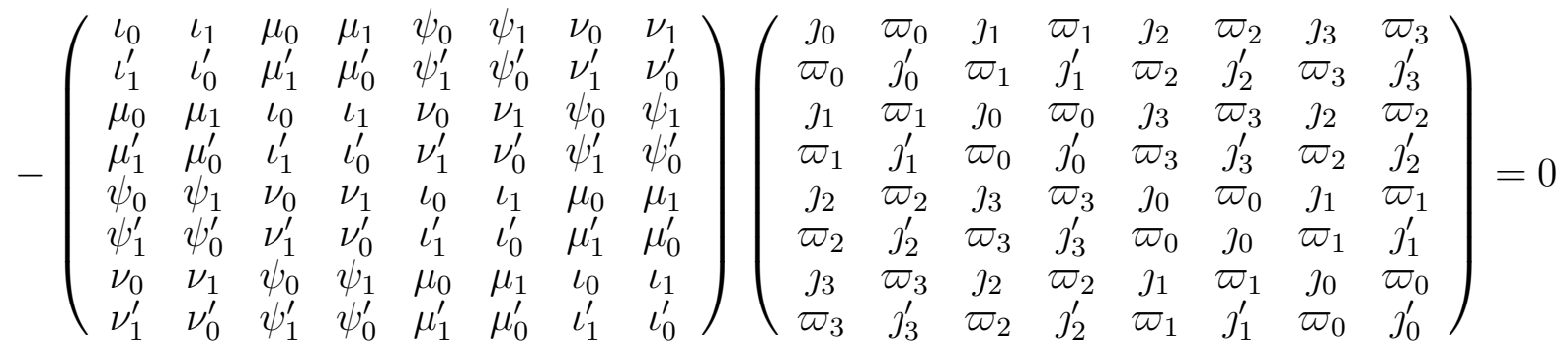

Where the matrices are defined below.

$\iota_{0}=\operatorname{circ}\left(t_{0}, t_{1}, t_{2}, t_{3}, \ldots . ., t_{2^{n}-1}\right), \iota_{1}=\operatorname{circ}\left(u_{0}, u_{1}, u_{2}, u_{3}, \ldots . ., u_{2^{n}-1}\right)$,

$\mu_{0}=\operatorname{circ}\left(v_{0}, v_{5}, v_{2}, v_{7}, \ldots ., v_{2^{n}-1}\right), \mu_{1}=\operatorname{circ}\left(w_{0}, w_{5}, w_{2}, w_{7}, \ldots . ., w_{2^{n}-1}\right)$,

$\psi_{0}=\operatorname{circ}\left(X_{0}, X_{1}, X_{2}, X_{3}, \ldots \ldots, X_{2^{n}-1}\right), \psi_{1}=\operatorname{circ}\left(z_{0}, z_{1}, z_{2}, z_{3}, \ldots \ldots, z_{2^{n}-1}\right)$,

$\nu_{0}=\operatorname{circ}\left(y_{0}, y_{1}, y_{2}, y_{3}, \ldots . ., y_{2^{n}-1}\right), \nu_{1}=\operatorname{circ}\left(s_{0}, s_{1}, s_{2}, s_{3}, \ldots . ., s_{2^{n}-1}\right)$,

$\iota_{0}^{\prime}=\operatorname{circ}\left(t_{0}, t_{1+z}, t_{2}, t_{3+z}, \ldots . ., t_{2^{n}+z-1}\right), \iota_{1}^{\prime}=\operatorname{circ}\left(u_{0}, u_{1+z}, u_{2}, u_{3+z}, \ldots ., u_{2^{n}+z-1}\right)$,

$\mu_{0}^{\prime}=\operatorname{circ}\left(v_{0}, v_{1+z}, v_{2}, v_{3+z}, \ldots . ., v_{2^{n}+z-1}\right), \mu_{1}^{\prime}=\operatorname{circ}\left(w_{0}, w_{1+z}, w_{2}, w_{3+z}, \ldots . ., w_{2^{n}+z-1}\right)$,

$\psi_{0}^{\prime}=\operatorname{circ}\left(X_{0}, X_{1+z}, X_{2}, X_{3+z}, \ldots \ldots, X_{2^{n}+z-1}\right), \psi_{1}^{\prime}=\operatorname{circ}\left(z_{0}, z_{1+z^{\prime}}, z_{2}, z_{3+z^{\prime}}, \ldots ., z_{2^{n}+z^{\prime}-1}\right)$,

$\nu_{0}^{\prime}=\operatorname{circ}\left(y_{0}, y_{1+z}, y_{2}, y_{3+z}, \ldots \ldots, y_{2^{n}+z-1}\right), \nu_{1}^{\prime}=\operatorname{circ}\left(s_{0}, s_{1+z}, s_{2}, s_{3+z}, \ldots ., s_{2^{n}+z-1}\right)$,

$\jmath_{0}=\operatorname{circ}\left(\curlywedge_{0}, \curlywedge_{1}, \curlywedge_{2}, \curlywedge_{3}, \ldots . . \curlywedge_{2^{n}-1}\right), \varpi_{0}=\operatorname{circ}\left(\curlyvee_{0}, \curlyvee_{1}, \curlyvee_{2}, \ldots, \curlyvee_{z-1}, \curlyvee_{0}, \curlyvee_{1}, \curlyvee_{2}, \ldots, \curlyvee_{z-1}\right)$,

$\jmath_{1}=\operatorname{circ}\left(e_{0}, e_{1}, e_{2}, e_{3}, \ldots ., e_{2^{n}-1}\right), \varpi_{1}=\operatorname{circ}\left(f_{0}, f_{1}, f_{2}, \ldots, f_{z-1}, f_{0}, f_{1}, f_{2}, \ldots, f_{z-1}\right)$,

$\jmath_{2}=\operatorname{circ}\left(g_{0}, g_{1}, g_{2}, g_{3}, \ldots . ., g_{2^{n}-1}\right), \varpi_{2}=\operatorname{circ}\left(h_{0}, h_{1}, h_{2}, \ldots, h_{z-1}, h_{0}, h_{1}, h_{2}, \ldots, h_{z-1}\right)$,

$\jmath_{3}=\operatorname{circ}\left(I_{0}, I_{1}, I_{2}, I_{3}, \ldots ., I_{2^{n}-1}\right), \varpi_{3}=\operatorname{circ}\left(J_{0}, J_{1}, J_{2}, \ldots, J_{z-1}, J_{0}, J_{1}, J_{2}, \ldots, J_{z-1}\right)$,

$J_{0}^{\prime}=\operatorname{circ}\left(\curlywedge_{0}, \curlywedge_{1+z}, \curlywedge_{2}, \curlywedge_{3+z}, \ldots . ., \curlywedge_{2^{n}+z-1}\right), \jmath_{1}^{\prime}=\operatorname{circ}\left(e_{0}, e_{1+z}, e_{2}, e_{3+z}, \ldots . ., e_{2^{n}+z-1}\right)$,

$\jmath_{2}^{\prime}=\operatorname{circ}\left(g_{0}, g_{1+z}, g_{2}, g_{3+z}, \ldots \ldots, g_{2^{n}+z-1}\right)$ and $\jmath_{3}^{\prime}=\operatorname{circ}\left(I_{0}, I_{1+z}, I_{2}, I_{3+z}, \ldots . ., I_{2^{n}+z-1}\right)$.

Therefore,

then this implies that

$$
\Gamma(\omega) \Gamma(v)-\Gamma(v) \Gamma(\omega)=0
$$

$\curlywedge_{1}=\curlywedge_{1+z}, \curlywedge_{3}=\curlywedge_{3+z}, \ldots . ., \curlywedge_{z-1}=\curlywedge_{2^{n-1}}, e_{1}=e_{1+z}, e_{3}=e_{3+z}, \ldots ., e_{z-1}=e_{2^{n-1}}$, $g_{1}=g_{1+z}, g_{3}=g_{3+z}, \ldots . ., g_{z-1}=g_{2^{n-1}}$ and $I_{1}=I_{1+z}, I_{3}=I_{3+z}, \ldots . ., I_{z-1}=I_{2^{n-1}}$ which gives the result.

3.7. The Structure of $Z\left(V_{*}\left(F_{2^{k}}\left(\left(M_{2^{n+1}} \times C_{2}\right) \times C_{2}\right)\right)\right)$.

Lemma 3.8. $Z(V)$ is a unitary unit subgroup. 
Proof. Consider an arbitrary element $m$ from center of $\mathrm{V}$, then

$$
\Gamma(m)=\left(\begin{array}{cccccccc}
\jmath_{0} & \ell_{0} & \jmath_{1} & \ell_{1} & \jmath_{2} & \ell_{2} & \jmath_{3} & \ell_{3} \\
\ell_{0} & \jmath_{0} & \ell_{1} & \jmath_{1} & \ell_{2} & \jmath_{2} & \ell_{3} & \jmath_{3} \\
\jmath_{1} & \ell_{1} & \jmath_{0} & \ell_{0} & \jmath_{3} & \ell_{3} & \jmath_{2} & \ell_{2} \\
\ell_{1} & \jmath_{1} & \ell_{0} & \jmath_{0} & \ell_{3} & \jmath_{3} & \ell_{2} & \jmath_{2} \\
\jmath_{2} & \ell_{2} & \jmath_{3} & \ell_{3} & \jmath_{0} & \ell_{0} & \jmath_{1} & \ell_{1} \\
\ell_{2} & \jmath_{2} & \ell_{3} & \jmath_{3} & \ell_{0} & \jmath_{0} & \ell_{1} & \jmath_{1} \\
\jmath_{3} & \ell_{3} & \jmath_{2} & \ell_{2} & \jmath_{1} & \ell_{1} & \jmath_{0} & \ell_{0} \\
\ell_{3} & \jmath_{3} & \ell_{2} & \jmath_{2} & \ell_{1} & \jmath_{1} & \ell_{0} & \jmath_{0}
\end{array}\right)
$$

where the above circulant matrices are defined below

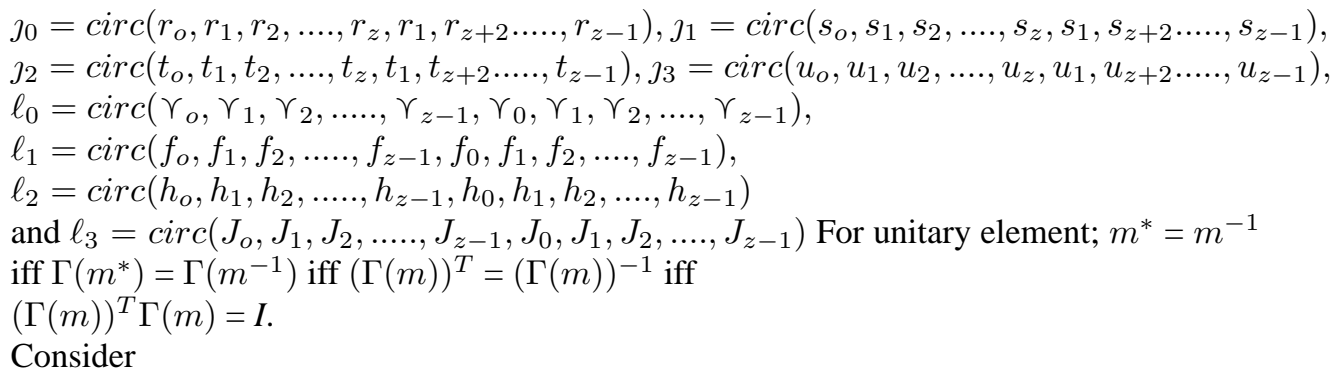

\section{Consider}

$$
\begin{aligned}
&(\Gamma(m))(\Gamma(m))^{T}=\left(\begin{array}{cccccccc}
\jmath_{0} & \ell_{0} & \jmath_{1} & \ell_{1} & \jmath_{2} & \ell_{2} & \jmath_{3} & \ell_{3} \\
\ell_{0} & \jmath_{0} & \ell_{1} & \jmath_{1} & \ell_{2} & \jmath_{2} & \ell_{3} & \jmath_{3} \\
\jmath_{1} & \ell_{1} & \jmath_{0} & \ell_{0} & \jmath_{3} & \ell_{3} & \jmath_{2} & \ell_{2} \\
\ell_{1} & \jmath_{1} & \ell_{0} & \jmath_{0} & \ell_{3} & \jmath_{3} & \ell_{2} & \jmath_{2} \\
\jmath_{2} & \ell_{2} & \jmath_{3} & \ell_{3} & \jmath_{0} & \ell_{0} & \jmath_{1} & \ell_{1} \\
\ell_{2} & \jmath_{2} & \ell_{3} & \jmath_{3} & \ell_{0} & \jmath_{0} & \ell_{1} & \jmath_{1} \\
\jmath_{3} & \ell_{3} & \jmath_{2} & \ell_{2} & \jmath_{1} & \ell_{1} & \jmath_{0} & \ell_{0} \\
\ell_{3} & \jmath_{3} & \ell_{2} & \jmath_{2} & \ell_{1} & \jmath_{1} & \ell_{0} & \jmath_{0}
\end{array}\right)\left(\begin{array}{ccccccccc}
\jmath_{0} & \ell_{0} & \jmath_{1} & \ell_{1} & \jmath_{2} & \ell_{2} & \jmath_{3} & \ell_{3} \\
\ell_{0} & \jmath_{0} & \ell_{1} & \jmath_{1} & \ell_{2} & \jmath_{2} & \ell_{3} & \jmath_{3} \\
\jmath_{1} & \ell_{1} & \jmath_{0} & \ell_{0} & \jmath_{3} & \ell_{3} & \jmath_{2} & \ell_{2} \\
\ell_{1} & \jmath_{1} & \ell_{0} & \jmath_{0} & \ell_{3} & \jmath_{3} & \ell_{2} & \jmath_{2} \\
\jmath_{2} & \ell_{2} & \jmath_{3} & \ell_{3} & \jmath_{0} & \ell_{0} & \jmath_{1} & \ell_{1} \\
\ell_{2} & \jmath_{2} & \ell_{3} & \jmath_{3} & \ell_{0} & \jmath_{0} & \ell_{1} & \jmath_{1} \\
\jmath_{3} & \ell_{3} & \jmath_{2} & \ell_{2} & \jmath_{1} & \ell_{1} & \jmath_{0} & \ell_{0} \\
\ell_{3} & \jmath_{3} & \ell_{2} & \jmath_{2} & \ell_{1} & \jmath_{1} & \ell_{0} & \jmath_{0}
\end{array}\right) \\
&=\left(\begin{array}{cccccccccc}
\alpha & 0 & 0 & 0 & 0 & 0 & 0 & 0 \\
0 & \alpha & 0 & 0 & 0 & 0 & 0 & 0 \\
0 & 0 & \alpha & 0 & 0 & 0 & 0 & 0 \\
0 & 0 & 0 & \alpha & 0 & 0 & 0 & 0 \\
0 & 0 & 0 & 0 & \alpha & 0 & 0 & 0 \\
0 & 0 & 0 & 0 & 0 & \alpha & 0 & 0 \\
0 & 0 & 0 & 0 & 0 & 0 & \alpha & 0 \\
0 & 0 & 0 & 0 & 0 & 0 & 0 & \alpha
\end{array}\right)
\end{aligned}
$$

where, $\alpha=\jmath_{0}^{2}+\ell_{0}^{2}+\jmath_{1}^{2}+\ell_{1}^{2}+\jmath_{2}^{2}+\ell_{2}^{2}+\jmath_{3}^{2}+\ell_{3}^{2}$ by using Theorem 2.3, we have $\alpha=\jmath_{0}^{2}+\ell_{0}^{2}+\jmath_{1}^{2}+\ell_{1}^{2}+\jmath_{2}^{2}+\ell_{2}^{2}+\jmath_{3}^{2}+\ell_{3}^{2}=I$. 


$$
=\left(\begin{array}{llllllll}
I & 0 & 0 & 0 & 0 & 0 & 0 & 0 \\
0 & I & 0 & 0 & 0 & 0 & 0 & 0 \\
0 & 0 & I & 0 & 0 & 0 & 0 & 0 \\
0 & 0 & 0 & I & 0 & 0 & 0 & 0 \\
0 & 0 & 0 & 0 & I & 0 & 0 & 0 \\
0 & 0 & 0 & 0 & 0 & I & 0 & 0 \\
0 & 0 & 0 & 0 & 0 & 0 & I & 0 \\
0 & 0 & 0 & 0 & 0 & 0 & 0 & I
\end{array}\right)
$$

Hence $m \in \mathrm{Z}(\mathrm{V})$ is an element of $V_{*}\left(F_{2^{k}}\left(\left(M_{2^{n+1}} \times C_{2}\right) \times C_{2}\right)\right)$. Thus $\mathrm{Z}(\mathrm{V}) \subset V_{*}\left(F_{2^{k}}\left(\left(M_{2^{n+1}} \times\right.\right.\right.$ $\left.\left.\left.C_{2}\right) \times C_{2}\right)\right)$.

Theorem 3.9. $Z\left(V_{*}\left(F_{2^{k}}\left(\left(M_{2^{n+1}} \times C_{2}\right) \times C_{2}\right)\right)\right) \cong C_{2}^{\left(2^{n+1}+2^{n} .3\right) k}$, where $Z\left(V_{*}\left(F_{2^{k}}\left(\left(M_{2^{n+1}} \times\right.\right.\right.\right.$ $\left.\left.\left.C_{2}\right) \times C_{2}\right)\right)$ ). is center of unitary unit subgroup $V_{*}\left(F_{2^{k}}\left(\left(M_{2^{n+1}} \times C_{2}\right) \times C_{2}\right)\right)$.

Proof. Let us denote again $V=V_{*}\left(F_{2^{k}}\left(\left(M_{2^{n+1}} \times C_{2}\right) \times C_{2}\right)\right)$. and recall that $Z(V)=$ $\left.\left.\left\{r_{0}((1,1), 1)\right\}+r_{1}\{(\psi, 1), 1)+\left(\left(\psi^{z+1}, 1\right), 1\right)\right\}+r_{3}\left\{\left(\psi^{3}, 1\right), 1\right)+\left(\left(\psi^{z+3}, 1\right), 1\right)\right\}+\ldots .+$ $r_{z-1}\left\{\left(\left(\psi^{z-1}, 1\right), 1\right)+\left(\left(\psi^{2^{n}-1}, 1\right), 1\right)\right\}+r_{2}\left(\left(\psi^{2}, 1\right), 1\right)+r_{4}\left(\left(\psi^{4}, 1\right), 1\right)+\ldots .+r_{2^{n}-2}\left(\left(\psi^{2^{n}-2}, 1\right), 1\right)+$ $\left.\left\{s_{0}((1, \iota), 1)+s_{1}\{(\psi, \iota), 1)+\left(\left(\psi^{z+1}, \iota\right), 1\right)\right\}+s_{3}\left\{\left(\psi^{3}, \iota\right), 1\right)+\left(\left(\psi^{z+3}, \iota\right), 1\right)\right\}+\ldots .+$ $s_{z-1}\left\{\left(\left(\psi^{z-1}, \iota\right), 1\right)+\left(\left(\psi^{2^{n}-1}, \iota\right), 1\right)\right\}+s_{2}\left(\left(\psi^{2}, \iota\right), 1\right)+s_{4}\left(\left(\psi^{4}, \iota\right), 1\right)+\ldots+s_{2^{n}-2}\left(\left(\psi^{2^{n}-2}, \iota\right), 1\right)$ $\left.\left\{t_{0}((1, \iota), \iota)+t_{1}\{(\psi, \iota), \iota)+\left(\left(\psi^{z+1}, \iota\right), \iota\right)\right\}+t_{3}\left\{\left(\psi^{3}, \iota\right), \iota\right)+\left(\left(\psi^{z+3}, \iota\right), \iota\right)\right\}+\ldots .+$ $t_{z-1}\left\{\left(\left(\psi^{z-1}, \iota\right), \iota\right)+\left(\left(\psi^{2^{n}-1}, \iota\right), \iota\right)\right\}+t_{2}\left(\left(\psi^{2}, \iota\right), \iota\right)+s_{4}\left(\left(\psi^{4}, \iota\right), \iota\right)+\ldots+t_{2^{n}-2}\left(\left(\psi^{2^{n}-2}, \iota\right), \iota\right)$ $\left.\left\{u_{0}((1,1), \iota)+u_{1}\{(\psi, 1), \iota)+\left(\left(\psi^{z+1}, 1\right), \iota\right)\right\}+u_{3}\left\{\left(\psi^{3}, 1\right), \iota\right)+\left(\left(\psi^{z+3}, 1\right), \iota\right)\right\}+\ldots . .+$ $u_{z-1}\left\{\left(\left(\psi^{z-1}, 1\right), \iota\right)+\left(\left(\psi^{2^{n}-1}, 1\right), \iota\right)\right\}+u_{2}\left(\left(\psi^{2}, 1\right), \iota\right)+u_{4}\left(\left(\psi^{4}, 1\right), \iota\right)+\ldots .+u_{2^{n}-2}\left(\left(\psi^{2^{n}-2}, 1\right), \iota\right)+$ $\sum_{\dot{a}=0}^{z-1} \curlyvee_{\dot{a}}\left\{\left(\left(\psi^{\dot{a}} \lambda, 1\right), 1\right)+\left(\left(\psi^{\dot{a}+z} \lambda, 1\right), 1\right)\right\}+\sum_{\dot{a}=0}^{z-1} f_{\dot{a}}\left\{\left(\left(\psi^{\dot{a}} \lambda, \iota\right), 1\right)\left(\left(\psi^{\dot{a}+z} \lambda, \iota\right), 1\right)\right\}+$ $\sum_{\dot{a}=0}^{z-1} h_{\dot{a}}\left\{\left(\left(\psi^{\dot{a}} \lambda, 1\right), \iota\right)+\left(\left(\psi^{\dot{a}+z} \lambda, 1\right), \iota\right)\right\}+\sum_{\dot{a}=0}^{z-1} J_{\dot{a}}\left\{\left(\left(\psi^{\dot{a}} \lambda, \iota\right), \iota\right)+\left(\left(\psi^{\dot{a}+z} \lambda, \iota\right), \iota\right)\right\}$ where, $r_{o}=1+r_{2}+r_{4}+\ldots . .++r_{2^{n}-2}+s_{0}+s_{2}+s_{4}+\ldots . .+s_{2^{n}-2}+t_{0}+t_{2}+$ $t_{4}+\ldots . .+t_{2^{n}-2}+u_{0}+u_{2}+u_{4}+\ldots . .+u_{2^{n}-2 . .}$ From lemma 3.2 we have $\mathrm{Z}(\mathrm{V}) \subset$ $V_{*}\left(F_{2^{k}}\left(\left(M_{2^{n+1}} \times C_{2}\right) \times C_{2}\right)\right)$. But $V=V_{*}\left(F_{2^{k}}\left(\left(M_{2^{n+1}} \times C_{2}\right) \times C_{2}\right)\right)$. This implies that $\mathrm{Z}(\mathrm{V})=\mathrm{Z}\left(V_{*}\left(F_{2^{k}}\left(\left(M_{2^{n+1}} \times C_{2}\right) \times C_{2}\right)\right)\right)$. Therefore $\mathrm{Z}\left(V_{*}\left(F_{2^{k}}\left(\left(M_{2^{n+1}} \times C_{2} \times C_{2}\right)\right)\right) \cong\right.$ $C_{2}^{\left(2^{n+1}+2^{n} \cdot 3\right) k}$.

\section{ACKNOWLEDGMENT}

The authors are thankful to the anonymous referees for their careful reading of the manuscript and providing their useful comments which have been encompassed in the final version of the manuscript.

\section{REFERENCES}

[1] V. Bovdi and L. G. Kovacs, Unitary units in modular group algebras, Manuscripta Mathematica, 84, No .1 (1994) 57-72.

[2] V. Bovdi and A. L. Rosa, On the order of the unitary subgroup of a modular group algebra, Communications in Algebra, 28, No. 4 (2000) 1897-1905.

[3] A. A. Bovdi and A. A. Sakach, Unitary subgroup of the multiplicative group of a modular group algebra of a finite abelian p-group, Mathematical Notes of the Academy of Sciences of the USSR, 45, No.6 (1989)445450.

[4] P. J. Davis,Circulant matrices. John Wiley and Sons, New York(1979). 
[5] J. Gildea, On the order of $U\left(F_{p^{k}} D_{2 p^{m}}\right)$, International Journal of Pure and Applied Mathematics, 46, No. 2 (2008) 267-272.

[6] T. Hurley, Group rings and rings of matrices, International Journal of Pure and Applied Mathematics, 31, No.3 (2006) 319-335.

[7] C. P. Milies, S. K. Sehgal, and S. Sehgal, An introduction to group rings, Springer Science and Business Media. 1 (2002).

[8] Z. Raza, and M. Ahmad, On the unitary units of the group algebra $F_{2} m M_{16}$, Journal of Algebra and its Applications, 12, No.08 (2013) 1350059.

[9] Z. Raza and M. Ahmad, Structure of the unitary subgroup of the group algebra $F_{2^{n}}(Q D)_{16}$, Journal of Algebra and Its Applications, 17, No. 4 (2018) 1850060.

[10] R. Sandling, Units in the modular group algebra of a finite abelian p-group, Journal of Pure and Applied Algebra, 33, No.3 (1984) 337-346.

[11] R. Sandling, Presentations for unit groups of modular group algebras of groups of order 16, Mathematics of Computation, 59, No .200 (1992) 689-701. 